\title{
Syncope Diagnosed by Inducible Sustained Ventricular Tachycardia
}

Amit Vira, MD

\section{INTRODUCTION}

Syncope is a common complaint in the emergency department (ED), accounting for approximately $3 \%$ of all ED visits. ${ }^{1}$ Although most causes of syncope are benign and self-limited, others are associated with significant morbidity and mortality.

\section{CASE PRESENTATION}

An 85-year-old male with a history of coronary artery disease status post prior coronary artery bypass grafting, moderate to severe aortic stenosis, hypertension, and stage IIIA chronic kidney disease presents to emergency room after abrupt onset of unwitnessed syncope. He reports exercising on an elliptical machine at home and falling on the floor. He denies any prodrome symptoms of chest discomfort, shortness of breath, palpitations, flushing, feeling cold or clammy, visual disturbances, nausea or vomiting. He believes the episode was brief and woke up within seconds feeling completely back to his baseline. He denies any confusion, bladder or bowel incontinence, or physical trauma immediately after the syncope. He reports waking up that morning feeling well, eating a normal breakfast and staying well-hydrated.

His medications include aspirin, atorvastatin, amlodipine, and rampiril. He has no known tobacco, alcohol, or illicit drug use.
Physical exam: Temperature $98.4^{\circ} \mathrm{F}$, Blood pressure $129 / 62$, Heart rate 82 , Respiratory rate 16 , saturating $99 \%$ on ambient air. Orthostatic vitals were negative. Cardiac exam revealed a regular 3/6 mid-peaking systolic ejection murmur with preservation of $\mathbf{S} 2$ at the cardiac base. No jugular venous distention. Lungs were clear to auscultation and the abdomen exam was soft, non-tender, with normoactive bowel sounds. Extremities were warm with +2 equal peripheral pulses. Neurologic exam was non-focal with normal gait.

Laboratory Studies: Metabolic panel was significant for a creatinine of $1.4 \mathrm{mg} / \mathrm{dL}$ (at baseline) with the remainder of the electrolytes being normal. Complete blood count was significant for a hemoglobin level of $12.3 \mathrm{~g} / \mathrm{dL}$ (at baseline). His serial cardiac enzymes were negative.

EKG showed normal sinus rhythm, normal QRS duration, corrected QT of $455 \mathrm{~ms}$, incomplete right bundle branch and multiple premature ventricular complexes. An echocardiogram revealed low normal right and left ventricular function with inferoapical akinesis consistent with an old infarcted scar and moderate aortic stenosis with a mean gradient of $30 \mathrm{mmHg}$ and valve area of 1.0 $\mathrm{cm}^{2}$, which was unchanged from a prior echocardiogram. 24-hour telemetry revealed multiple premature ventricular complexes and short runs of non-sustained ventricular tachycardia (Figure 1).

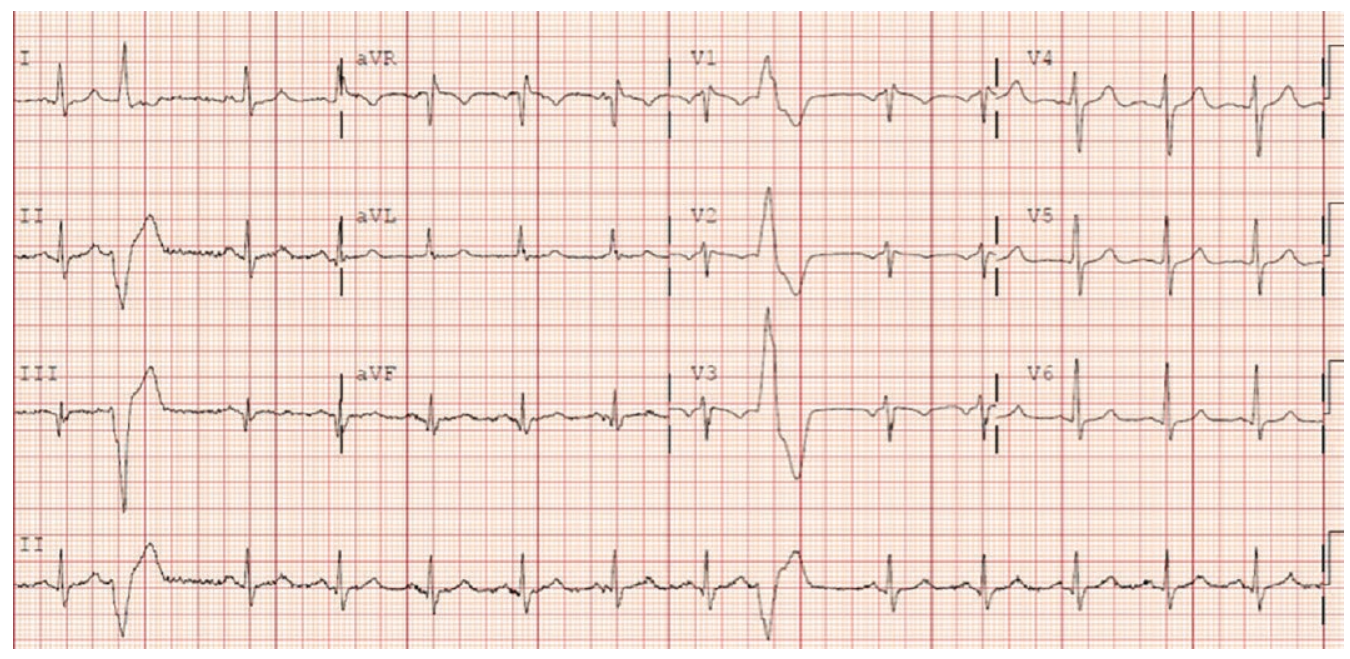

Figure 1. Patient's continuous cardiac monitoring revealed multiple premature ventricular complexes (seen on EKG) and short runs of non-sustained ventricular tachycardia. 


\section{OUTCOME}

Given his abrupt exertional syncope during exercise without prodrome, cardiac syncope was suspected. EKG showed evidence of conduction disease and significant ventricular ectopy. His overall echocardiogram was unchanged from prior and showed focal scar. Given this constellation of signs and symptoms, a ventricular tachyarrhythmias was suspected as the cause. Electrophysiologic study was recommended and revealed an easily inducible sustained monomorphic ventricular tachycardia. The ventricular tachycardia showed a right bundle branch pattern with left superior axis morphology consistent with an inferoapical site with the likely origin being from the scar from his prior myocardial infarction as seen on echocardiogram. Given these findings, an implantable cardioverter defibrillator was recommended for secondary prevention.

\section{DISCUSSION}

Syncope or "true syncope" is the sudden and transient loss of consciousness and postural tone attributable to inadequate cerebral blood flow. In comparison, pre-syncope refers to near loss of consciousness and occurs more commonly than syncope. However, both syncope and pre-syncope should be considered in the same disease spectrum.

There are many causes for transient loss of consciousness (Table 1). Non-syncopal causes of transient loss of consciousness include seizure disorders, concussions, intoxications, metabolic disturbances, and conversion disorders. Distinguishing these conditions from true syncope is challenging, but it is crucial in order to determine appropriate management.

The differential diagnosis of true syncope is broad, and management focuses on treating the underlying cause. Below are the most common causes of syncope seen in emergency rooms.

1. Neurally mediated syncope is one of the most frequent etiologies of syncope and is often referred to as vasovagal syncope. ${ }^{2}$ It is characterized by peripheral vasodilation and hypotension, along with bradycardia which is believed to be due an increase in parasympathetic tone and concomitant inhibition of sympathetic outflow. ${ }^{3}$ The hypotension can lead to loss of consciousness if severe or pre-syncope if less severe. A wide variety of stimuli can trigger this reflex, the most common stimulus being orthostatic stress. Other triggers include prolonged standing, crowded places, or unpleasant sight, smell, or pain. Typically, one experiences nausea, lightheadedness, a feeling of warmth, and pallor before losing consciousness.

\section{Table 1: Common Causes of Syncope}

Neurally mediated

Vasovagal (emotional stress, pain, standing)

Orthostatic hypotension

Drug induced (alpha-blockers, vasodilators)

Hypovolemia (dehydration, blood loss)

Diabetic neuropathy

Parkinson's disease

Dementia

Aging

Cardiac arrhythmias

Supraventricular tachyarrhythmias

Ventricular tachyarrhythmias

Torsade de pointes

Sinus bradycardia

High-grade atrioventricular blocks

Pacemaker malfunction

Cardiac non-arrhythmias

Advanced cardiomyopathy

Aortic stenosis

Hypertrophic obstructive cardiomyopathy

Pulmonary embolism

Pulmonary stenosis

Pericardial tamponade

Acute myocardial infarction

Aortic dissection

Table 2: High Risk Criteria Requiring Prompt Hospitalization

Severe structural or coronary artery disease

Heart failure or previous myocardial infarction

Clinical or ECG features suggesting arrhythmic syncope

Exertional syncope

Palpitations preceding syncope

Family history of sudden cardiac death

Non-sustained ventricular tachycardia

Evidence of atrioventricular block

Bifascicular-block

Pre-excited QRS complex

Prolonged or short QT interval

Brugada pattern 
2. Orthostatic hypotension is characterized by an inadequate physiologic response to postural changes in blood pressure that decreases cerebral perfusion. A wide variety of conditions can cause postural hypotension, with two major mechanisms including autonomic failure and volume depletion. Autonomic failure is further subdivided into intrinsic and extrinsic. Intrinsic causes include neurodegenerative diseases (ie Parkinson's dementia) or neuropathies (i.e. diabetes) while extrinsic causes include medications (i.e. alpha-blockers, phosphodiesterase inhibitors, or vasodilators). ${ }^{4}$ In comparison, severe intravascular volume depletion (i.e. dehydration or blood loss) can lead to syncope due to functional failure of the system despite having normal functioning autonomic reflexes. Common symptoms of orthostatic hypotension include dizziness, lightheadedness, weakness, fatigue, or nausea.

3. Cardiac syncope is often the most concerning as it can occur without warning leading to major morbidity and mortality. It often occurs either with conduction system disease (i.e. brady- and tachyarrhythmias) or in the presence of structural abnormalities such as valvular disease or cardiomyopathies. Bradyarrhythmia can develop from natural pacemaker dysfunction or the development of heart block. Tachyarrythmias can be subdivided into either supraventricularorventricularin origin. Supraventricular tachycardia usually causes palpitations but rarely causes syncope. In contrast, ventricular tachyarrythmias often cause syncope. Valvular heart disease, particularly aortic stenosis, can lead to syncope which most often occurs during exertion.

Typically, in older adults, cardiac syncope is often seen in patients with preexisting cardiovascular or structural heart disease and is often sudden without any prodrome. Common symptoms include palpitations, chest pain, and shortness of breath.

After a syncopal episode, the essential next step is to establish the etiology. For nearly all patients, the initial evaluation for syncope should include obtaining a comprehensive history, complete physical examination, and review of an electrocardiogram (ECG). A transthoracic echocardiogram is useful to evaluate for the presence and severity of structural heart disease if structural heart disease status is uncertain, after completion of a history, physical examination, and ECG. Additional diagnostic evaluation, if indicated, should be individualized based upon the suspected etiology of syncope.
Wherever the initial syncope evaluation leads, one must determine whether the affected individual needs in-hospital care for further evaluation and/or initiation of treatment. The primary factor determining whether the patient with presumed syncope should be hospitalized is the individual's immediate mortality risk. Patients can be classified as high risk (requiring admission), intermediate risk (admission is case-by-case), and low risk (can be outpatient). See table 2 for risk stratification..$^{5}$ In all cases, arrangements for prompt outpatient care is essential.

\section{KEY POINTS}

Have a high degree of suspicion for cardiac syncope when a patient presents with syncope without prodomal symptoms. A comprehensive history, complete physical examination, and review of an ECG is essential. High risk patients as identified by a history of preexisting cardiovascular disease or structural heart disease or baseline abnormal ECG should be hospitalized for further evaluation and treatment.

\section{REFERENCES}

1. Costantino G, Sun BC, Barbic F, et al. Syncope clinical management in the emergency department: A consensus from the first international workshop on syncope risk stratification in the emergency department. European Heart Journal 2016;37:1493-1498.

2. Sheldon RS, Grubb BP, Olshansky B, et al. 2015 Heart Rhythm Society Expert Consensus Statement on the Diagnosis and Treatment of Postural Tachycardia Syndrome, Inappropriate Sinus Tachycardia, and Vasovagal Syncope. Heart Rhythm 2015;12(6):e41-e63.

3. Mosqueda-Garcia R, Furlan R, Tank J. The Elusive Pathophysiology of Neurally Mediated Syncope. Circulation 2000;102:2898-2906.

4. Ricci F, De Caterina R, Fedorowski A. Orthostatic Hypotension: Epidemiology, Prognosis, and Treatment. JACC 2015;66(7):848-860.

5. Shen WK, Sheldon RS, Benditt DG, et al. 2017 ACC/AHA/HRS Guideline for the Evaluation and Management of Patients With Syncope: Executive Summary. JACC 2017;70(5):620-663. 